\title{
Valoración de las lesiones oculares producidas en los accidentes de tráfico.
} Assessment of ocular injuries due to traffic accidents.

\author{
JA. Menéndez'1, FJ. Pera² y R. Morcillo³
}

\section{RESUMEN}

Objetivo: Analizar cómo se realiza, desde el punto de vista pericial, la valoración de las lesiones y secuelas oculares de los accidentes de tráfico en nuestro medio.

Método: Hemos realizado un estudio retrospectivo de 127 informes sobre lesiones oculares realizados en la Sección de Oftalmología de la Clínica Médico Forense de Madrid durante cinco años (del 2002 al 2006), analizando las siguientes variables: la proporción que representan los accidentes de tráfico (ATF), el tipo de lesión producida en el ATF, la duración media de la baja, las secuelas más frecuentes y la puntuación total media asignada a estas secuelas oculares.

Resultados: Los ATF son la causa de la cuarta parte de las secuelas oculares que vemos. Las lesiones más frecuentemente producidas son traumatismos cráneo-encefálicos, contusiones oculares directas y esguinces cervicales. Hemos hallado un alto porcentaje de casos (22\%), en los que no encontramos lesiones objetivas que justifiquen las molestias alegadas por el lesionado, o bien estas no guardan relación evidente con el accidente de tráfico sufrido. Conclusiones: Que los ATF son una de las causas más frecuentes de secuelas oculares (diplopias, disminuciones de agudeza visual...), con una puntuación media de 15 puntos y una duración media de la baja de 71 días, hay un importante porcentaje de reclamaciones injustificadas. Considera necesario revisar el actual Baremo de la Ley $34 / 2003$, subsanando los errores y deficiencias que presenta, e incluyendo secuelas oculares típicamente postraumáticas que actualmente no recoge, para facilitar la valoración de las secuelas oculares.

Palabras clave: accidentes de tráfico, lesiones oculares, medicina forense, secuelas oculares, valoración del daño corporal.

Cuad Med Forense 2008; 14(51):25-33

\section{ABSTRACT}

Aim: To analyse how it is performed, from expert evidence view point, the assessment of ocular injuries and aftereffects due to traffic accidents in our area.

Method: We have accomplished a retrospective study of 127 reports on ocular injuries, carried out in the Ophthalmologic Section of the Medical Forensic Clinic of Madrid for five years -from 2002 to 2006-, analyzing the following variables: the proportion represented by traffic accidents (TAs); the kind of injury sustained in the TAs; the average length of sick leave; the most frequent after-effects; and the overall average punctuation given to these ocular after-effects.

Results: TAs represent the cause of a quarter of the ocular after-effects we see. The most frequent injuries produced in an accident are cranioencephalic traumatisms, direct ocular contusions, and cervical sprains. We have met a high percentage of cases (22\%) in which we have not found objective injuries that can justify the pains claimed by the injured, or rather the pains are not evidently connected with the TAs suffered.

Conclusions: TAs are one of the most frequent causes of ocular after-effects (diplopias, decreases of visual acuity...), with an average punctuation of 15 points and an average length of sick leave of 7 I days, existing an important percentage of unjustified claims. It is considered as necessary the revision of the present scale of the Law $34 / 2003$ rectifying errors and defects that it presents and including typically post-traumatic ocular after-effects, which are not registered presently, to facilitate the assessment of ocular after-effects.

Key words: traffic accidents, ocular injuries, forensic medicine, ocular after-effects, evaluation of corporal damage.

Este trabajo no ha sido subvencionado por entidades públicas, ni privadas

Correspondencia: Dr. José Antonio Menéndez de Lucas. c/ Fornells no 4, Ático B. 28230 Las Rozas (Madrid). Tfno. 6292737 57. Fax 916318276 e-mail: jamenendezdelucas@yahoo.es

1 Doctor en Medicina. Oftalmólogo de la Clínica Médico Forense de Madrid.

2 Doctor en Medicina. Director de la Clínica Médico Forense de Madrid.

3 Doctor en Medicina. FEA del Servicio de Oftalmología del Hospital Ramón y Cajal de Madrid. Fundación INCIVI. 


\section{INTRODUCCIÓN:}

La incidencia de lesiones oculares en los accidentes de tráfico (ATF) ha aumentado progresivamente en los últimos años. Los mecanismos de seguridad que incorporan actualmente los automóviles, concretamente los "air-bags" frontales, están contribuyendo de manera importante a este incremento, aunque debemos considerarlo como un inevitable tributo que tenemos que pagar frente a su indiscutible y demostrada eficacia en evitar la muerte de los ocupantes del vehículo cuando se produce un impacto frontal a alta velocidad. El uso del cinturón de seguridad ha demostrado ser el mecanismo más eficaz en la prevención de lesiones oculares en este tipo de accidentes [I].

Todo el que ha sufrido la desagradable experiencia de resultar lesionado en un accidente de tráfico, sabe perfectamente que nada nos puede compensar el daño sufrido como consecuencia de la distracción, imprudencia o negligencia de aquel en cuyo camino tuvimos la desgracia de cruzarnos, pero en un Estado de Derecho existen una serie de mecanismos jurídicos para exigirle que repare el daño causado. La finalidad de la responsabilidad penal es defender a la sociedad (retirada del permiso de conducir, multas,...), mientras que a través de la responsabilidad civil se intenta compensar al lesionado con una cantidad de dinero por el daño sufrido.

No resulta nada fácil fijar la cuantía de esta indemnización en cada caso concreto, por lo que pueden surgir discrepancias que deben resolver los Tribunales de Justicia. Los daños materiales producidos son sencillos de evaluar por el Juez; sin embargo la valoración de las secuelas físicas y psíquicas sufridas hace imprescindible el asesoramiento médico-legal del Magistrado.

Esta función pericial médica, que hasta hace poco tiempo realizaban de forma prácticamente exclusiva los médicos forenses, se ha ido desarrollando vertiginosamente en los últimos años hasta convertirse en una parcela con entidad propia dentro de la Medicina Legal y Forense, que se denomina Valoración del Daño Corporal (VDC). Actualmente participan en ella multitud de profesionales médicos: forenses, médicos de las compañías de seguros, expertos en valoración del daño corporal, especialistas en medicina legal, traumatólogos, neurólogos, oftalmólogos, etc... Consiste esencialmente en determinar las lesiones producidas en el accidente, el tiempo que ha tardado en curar el lesionado y las secuelas que le han quedado. Para realizar la valoración de estas secuelas habrá que encuadrarlas dentro de un baremo que, en el caso de las lesiones derivadas de accidentes de tráfico, tiene carácter vinculante y que figura en el anexo de la Ley 34/2.003 de Modificación y adaptación a la normativa comunitaria de la legislación de Seguros Privados.

El objetivo de este artículo es tratar de analizar cómo se realiza, desde el punto de vista pericial, la valoración de las lesiones y secuelas oculares de los accidentes de tráfico en nuestro medio. Trataremos de determinar el tipo de lesión que produce una secuela ocular, el tiempo medio de curación, las secuelas oftalmológicas más habituales y la puntuación media total que se les otorga. Analizaremos también las dificultades que pueden plantearse en la aplicación del baremo de la Ley 34/2003 en la práctica diaria, así como sus posibles soluciones.

\section{MATERIAL Y MÉTODO:}

Hemos realizado un estudio descriptivo retrospectivo de 127 informes periciales sobre valoración de lesiones oculares producidas como consecuencia de accidentes de tráfico. Estos informes fueron solicitados a la Sección de Oftalmología de la Clínica Médico Forense de Madrid, entre el I de enero de 2002 y el 3 I de diciembre de 2.006. El 5 I \% de la población fueron mujeres y el $49 \%$ hombres. La edad media de los lesionados fue 40,4I años (Des. Est. de I 8, I I años), siendo la edad máxima de 82 años y la mínima 8 años. En la figura I representamos la distribución de estos 
127 informes, en relación al número total de solicitudes en dicha Sección, durante este periodo de tiempo. En estos cinco años se solicitaron un total de 505 informes sobre valoración de lesiones oculares producidas como consecuencia de multitud de circunstancias: agresiones, accidentes laborales, de tráfico, denuncias por responsabilidad profesional, valoración de incapacidades laborales permanentes o minusvalías, etc...

Estos informes fueron solicitados siempre por un Juzgado de Instrucción de la Comunidad de Madrid, bien por iniciativa propia del Magistrado Instructor o bien a petición de las partes o del médico forense del Juzgado. El motivo de solicitar este tipo de informes al especialista en oftalmología de la Clínica suele ser la complejidad del caso, a la hora de realizar la exploración, o establecer la relación de causalidad entre la lesión o la secuela y el accidente de tráfico sufrido. En otros casos las partes pueden aportar informes contradictorios o no estar conformes con la valoración realizada por el forense del Juzgado Instructor, y solicitan informe del oftalmólogo de la Clínica Médico Forense. En todos los casos exploramos y valoramos personalmente al lesionado.

En los 127 informes analizados, hemos estudiado las siguientes variables:

- el número de informes sobre lesiones producidas por accidente de tráfico en relación al total de informes solicitados a la Sección de Oftalmología en estos cinco años.

- el tipo de lesión producida en el accidente de tráfico.

- la duración media de la baja.

- el tipo de secuelas oculares que se producen.

- la puntuación total media asignada a estas secuelas oculares.

\section{RESULTADOS:}

El número de informes sobre lesiones por ATF en relación al total de informes:

En la figura I se representa esta variable y en la parte inferior en color granate figura el valor porcentual que suponen los informes sobre lesiones oculares en relación al total de informes solicitados a la Clínica Médico Forense. El valor medio a lo largo de estos cinco años es del $26 \%$ de informes sobre lesiones oculares por ATF por año.

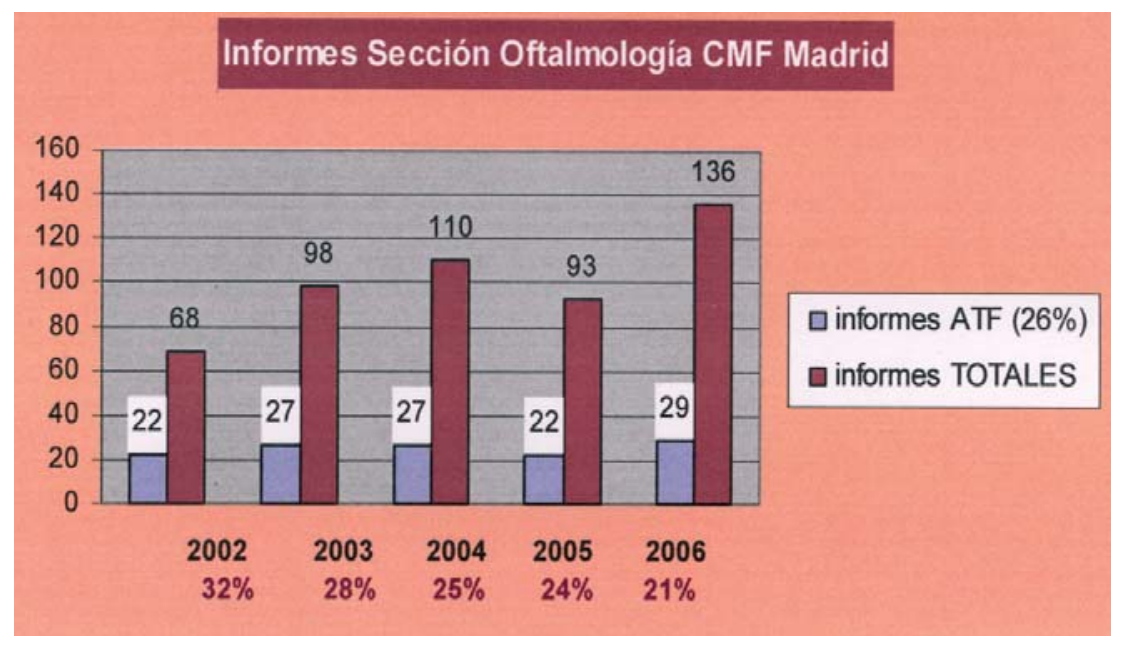

Figura 1. 


\section{Tipo de lesión producida en el accidente:}

Como podemos ver en la figura 2, las lesiones originales producidas en el accidente de tráfico y que se supone que ha ocasionado las secuelas oculares por las que se reclama una indemnización, en la mayoría de los casos (4I\%) son traumatismos craneoencefálicos (TCE), contusiones oculares directas (39\%), o esguinces cervicales (13\%). En menor proporción (5\%) se produce una lesión directa del párpado y hay algún caso $(2 \%)$ en los que se reclama sin que se produzca lesión alguna durante el accidente.

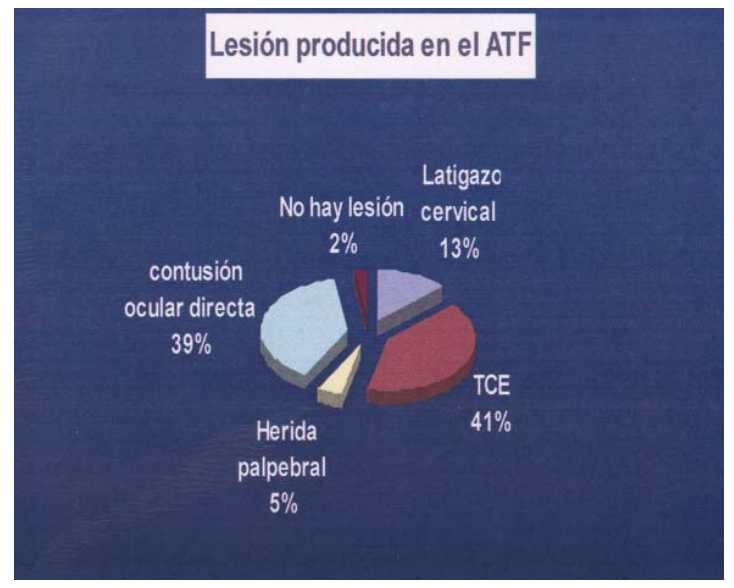

Figura 2.

Analizando el grupo más numeroso; el de los traumatismos craneoencefálicos (Figura 3), vimos que con frecuencia (37\%) producen una lesión postraumática de la musculatura extraocular que se traduce en una diplopia, también pueden reducir la agudeza visual (25\%), ocasionar lesiones campimétricas postraumáticas (12\%) o desencadenar un desprendimiento del vítreo posterior (8\%), pero encontramos además un porcentaje importante de casos (I8\%) en los que no existía secuela ocular o existía, pero no había datos objetivos que nos permitieran relacionarla etiológicamente con el TCE sufrido en el accidente.

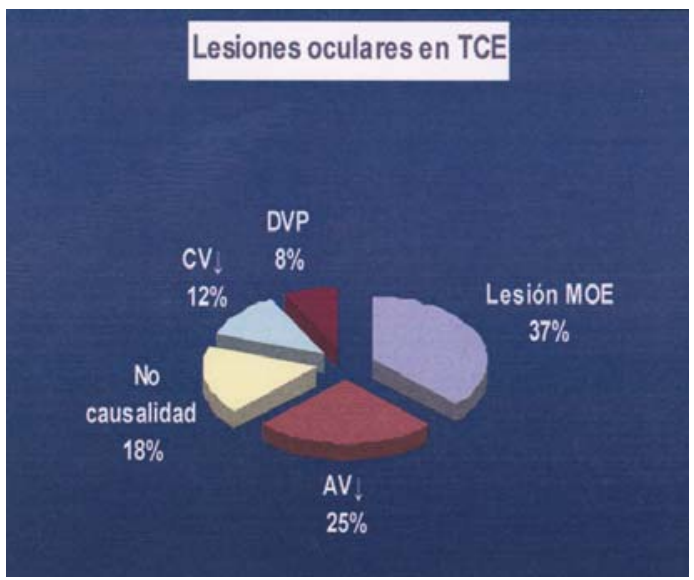

Figura 3. 
El grupo de las lesiones oculares consecutivas a esguinces cervicales, lo representamos en la figura 4. Encontramos que el porcentaje de casos en los que se reclama por una secuela inexistente o no relacionada con el accidente es aún mayor (41\%). Cuando en un esguince cervical se produce una lesión ocular, en la mayoría de los casos (4l\%) es un desprendimiento del vítreo posterior o se afecta un músculo extraocular produciendo visión doble (12\%) y encontramos que en el $6 \%$ de estos casos, se alega disminución de visión consecutiva al esguince.

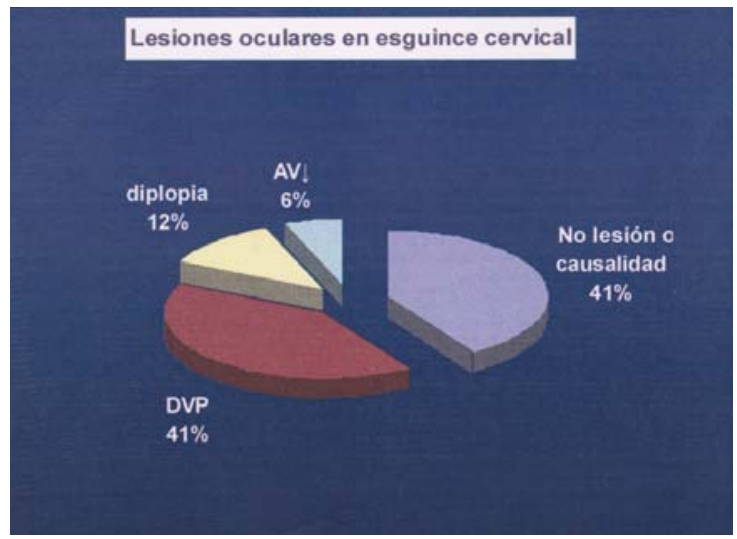

Figura 4.

En el grupo de lesiones oculares producidas por una contusión ocular directa (Figura 5), la secuela más habitual es una reducción de la agudeza visual. Ocurre en el 30\% de los casos y en la mitad de estos la reducción es tan importante que la visión del lesionado es inferior a 0. I0, por lo que se incluiría en el concepto de ceguera legal y lo englobamos en el epígrafe de amaurosis ( $14 \%$ ). Se produce una diplopia postraumática en muchos de estos casos (20\%), un desprendimiento del vítreo posterior (20\%) o una lesión permanente del polo anterior ( $4 \%$ ) y finalmente, hay otro grupo (16\%), en el que no encontramos una secuela ocular que podamos relacionar de forma objetiva o razonable con el accidente de tráfico sufrido.

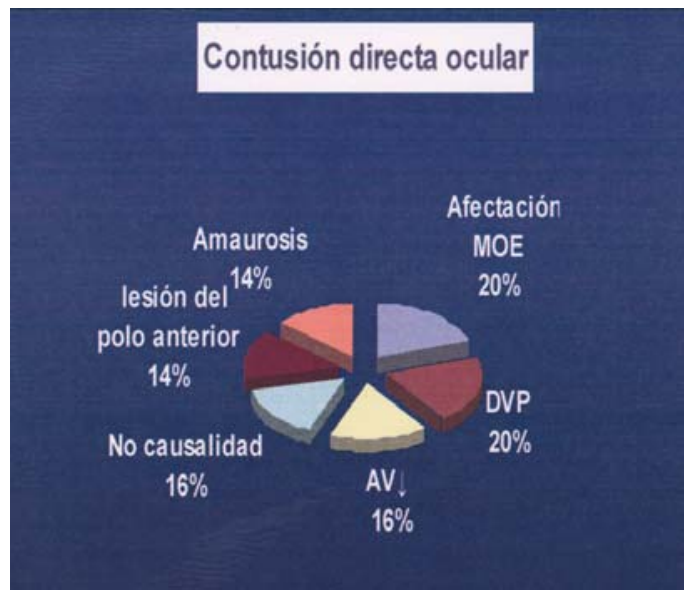

Figura 5. 


\section{La duración media de la baja:}

Pudimos analizar este parámetro al estar recogido en el $73 \%$ de los informes revisados. El periodo medio de duración de la baja por lesiones oculares es de 7I días impeditivos. Se llaman días impeditivos a lo que habitualmente entendemos como baja laboral.

\section{Las secuelas oculares producidas:}

Entendemos por secuela toda restricción anatómica o funcional de carácter permanente, es decir que persiste una vez agotado el tratamiento terapéutico y rehabilitador. Hemos agrupado las secuelas oculares en ocho categorías, que representamos en la figura 6.

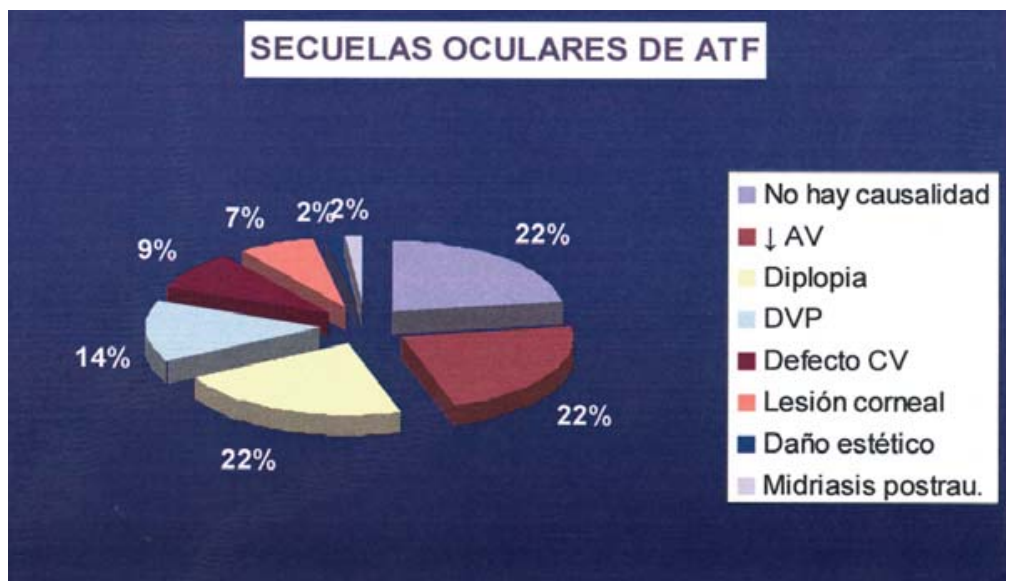

Figura 6.

Los tres grupos más frecuentes son las diplopias postraumáticas, los casos en los que se reduce la agudeza visual definitivamente y los casos en los que no encontramos una secuela ocular objetiva o la encontramos pero no consideramos que exista una relación de causalidad razonable con el accidente. Estos tres grupos comparten un porcentaje de incidencia del 22\%.

La secuela ocular más frecuente de los accidentes de tráfico, considerada de forma aislada, es el desprendimiento postraumático del vítreo posterior (DVP) que representa el 14\% de las secuelas oculares producidas en los accidentes de tráfico. En menor proporción se producen otras secuelas oculares: escotomas postraumáticos (9\%), secuelas corneales (erosión corneal recidivante, ojo seco postraumático,...) en el 7\%, o con menor frecuencia $(2 \%)$ se produce un daño estético o una lesión definitiva del iris (midriasis o discoria postraumática).

\section{Puntuación total de las secuelas oculares:}

En el $72 \%$ de los informes revisados, se recogía la puntuación total por las secuelas oculares. La media de estas puntuaciones totales analizadas ha sido I4,7| puntos. Para hacernos una idea aproximada de la que sería una indemnización media por secuelas oculares, podemos considerar que 15 puntos en un lesionado de una edad entre 20 y 40 años supondría un cantidad de 14.297 euros, ya que el valor del punto para esa franja de edad, conforme a la normativa vigente [2] es de $953,14 € /$ punto. 


\section{DISCUSIÓN:}

La mayoría de los trabajos publicados sobre traumatismos oculares están realizados en el ámbito asistencial, y los accidentes de tráfico ocasionan entre el 4 y el $12 \%$ de todos los traumatismos oculares atendidos en un servicio de urgencias hospitalario $[3,4,5]$. Dentro de los grandes traumatizados, la incidencia de lesiones oculares es baja salvo que existan fracturas faciales, en cuyo caso esta incidencia se incrementa 6,7 veces, produciéndose en la mayoría de los casos como consecuencia de accidentes de tráfico y siendo pacientes jóvenes habitualmente [6].

En un estudio prospectivo realizado sobre 67 pacientes hospitalizados en el Athens University Eye Clinic entre septiembre de 1.993 y diciembre de 1.996, por graves lesiones producidas como consecuencia de accidentes de tráfico, destaca que una mínima proporción (5,3\%) de los lesionados que viajaban en automóviles llevaba puesto el cinturón de seguridad y ninguno de los motoristas usaba casco. Las lesiones consistieron habitualmente en perforaciones oculares por fragmentos de cristales. Las secuelas fueron importantes, ya que la mitad de estos lesionados quedaron con una agudeza visual inferior a 0.5 . Concluye que, dado que los traumatismos oculares producidos en los ATF son una de las principales causas de pérdida o reducción importante de la visión, es necesario respetar las normas de seguridad y endurecer las leyes reguladoras de la obligatoriedad del uso del cinturón de seguridad. Nosotros encontramos menor proporción de lesionados con reducción importante de agudeza visual (22\%). Esta disparidad, podría explicarse por la menor gravedad de los lesionados en nuestra población, que no se limita a pacientes hospitalizados.

Tan sólo hemos encontrado una publicación sobre lesiones oculares por accidentes de tráfico dentro del ámbito pericial forense [7]. En este trabajo se compara el resultado de los potenciales visuales evocados (PVE) realizados en un grupo de 30 pacientes con secuelas oculares de accidentes de tráfico con otro grupo de igual número de pacientes, también con secuelas visuales, pero producidas por otra etiología. En los que habían sufrido un accidente de tráfico encuentran una proporción mucho más alta de traumatismos cráneo-encefálicos (TCE) y de parexias postraumáticas de pares craneales, que de diplopias, neuropatías ópticas postraumáticas o fracturas orbitarias.

Con frecuencia el lesionado alega una disminución de agudeza visual consecutiva al accidente y en algunos de estos casos, nos puede resultar difícil discriminar entre los casos reales y los simuladores. Las técnicas habituales de exploración de la agudeza visual en la práctica clínica son de tipo subjetivo, es decir, requieren de la colaboración del paciente. Las exploraciones subjetivas, en el ámbito pericial, tienen una utilidad muy relativa, por lo que estaría indicado utilizar pruebas de tipo objetivo como son los potenciales visuales evocados (PVE). Diferentes tipos de alteraciones visuales pueden mostrar diferentes patrones de alteración de los PVE (registro abolido, baja amplitud de la onda PI00, aumento de latencia de PI00,...).

Nosotros también hemos encontrado una proporción muy importante de lesionados que alegan secuelas oculares consecutivas a un TCE (4I\%) e incluso a un esguince cervical (I3\%).

El dato que nos ha resultado más sorprendente en la revisión realizada, ha sido el alto porcentaje de lesionados en los que no hallamos lesión orgánica que justifique las secuelas alegadas, o en los que encontramos lesiones que no son consecuencia del accidente. Esta situación ocurre en el I $8 \%$ de los TCE y en el $41 \%$ de los esguinces cervicales, que alegan secuelas oculares.

En algunos casos el lesionado puede creer de buena fe que sus molestias, al presentarse desde el mismo momento del accidente, guardan alguna relación etiopatogénica con este. Por el contrario, en otros muchos casos el lesionado inventa, exagera o atribuye secuelas al accidente siendo plenamente consciente de su falsedad, con lo que estaríamos ante un simulador [8]. La simulación es un fraude intencional, consciente y doloso, que como peritos médicos, tenemos la obligación de 
desenmascarar. Tratamos de evitar emplear el término "simulación" en nuestros informes, ya que como hemos visto, implica tener la certeza absoluta sobre la intencionalidad del sujeto, lo cual no siempre es posible. Consideramos mucho más correcto emplear el término de "pérdida visual funcional", que proponen otros autores [9] y nos parece menos comprometedor y "aséptico" desde el punto de vista pericial.

Es muy excepcional que un esguince cervical produzca secuelas visuales sin lesión ocular directa, aunque existen casos publicados que demuestran que puede darse esta posibilidad. Andonegui Navarro y Prat Madrazo, publican en 2004 un caso de retinopatía postraumática bilateral asociada a un síndrome de latigazo cervical [10]. En este caso se consideró la etiología vascular como la más probable. Según esta teoría, las lesiones se produjeron como consecuencia de un aumento de la presión intravascular que rompe la barrera hematorretinina, que se pudo evidenciar en las retinografías fluoresceínicas realizadas al poco tiempo del accidente, como un moteado hiperfluorescente bilateral. La valoración pericial de estos casos es difícil y requiere tener en cuenta una serie de circunstancias como son: el aspecto morfológico de la lesión, la proximidad en el tiempo entre el accidente y las primeras molestias, la existencia de "síntomas puente", además de poder excluir otros posibles mecanismos de producción de las lesiones. También resulta de mucha utilidad poder documentar las lesiones mediante pruebas de imagen (AFG, OCT, ECO,...).

En el grupo de lesionados que sufrieron contusión ocular directa también hemos encontrado una alta proporción (16\%) de casos que pretenden responsabilizar de sus molestias oculares al accidente sufrido. No es infrecuente encontrar lesionados que atribuyen el incremento de su miopía o la progresión de sus cataratas seniles a la uveítis postraumática que sufrieron tras el accidente y que curó sin secuelas objetivas.

La valoración pericial de este tipo de lesiones debe hacerse ajustándose a la normativa legal aplicable a estos casos: el Baremo contenido en el anexo de la Ley 34/2.003 [ I I], que como sabemos tiene carácter vinculante al realizar la valoración de las secuelas producidas como consecuencia de accidentes de tráfico. Hace unos años publicamos una crítica constructiva a este baremo [12], que introduce elementos en la valoración de las secuelas oculares que carecen de fundamento científico como pretender valorar un leucoma por el déficit campimétrico que ocasione, en lugar de hacerlo por el déficit de agudeza visual, o proponer añadir una serie de puntos adicionales a las lesiones postraumáticas del iris, "en caso de trastorno de la acomodación" [13], cuando como es sabido la acomodación se realiza mediante el músculo ciliar y no mediante el iris. Tampoco aclara definitivamente que la exploración de la agudeza visual que tiene trascendencia a la hora de valorar las secuelas de un traumatismo es para visión de lejos y con corrección. Este es un punto que plantea frecuentes dificultades prácticas que serían perfectamente evitables aclarándolo. Algo parecido ocurre con la forma de expresar la agudeza visual del lesionado; que debería ser en forma decimal, ya que es la que se emplea en la práctica clínica y por lo tanto la que figurará en los informes que aporta el lesionado, en lugar de hacerlo en forma de quebrado que actualmente resulta obsoleta. En este baremo no se recogen secuelas típicamente postraumáticas, como son una recesión angular que puede dar lugar a un glaucoma postraumático, o el desprendimiento del vítreo posterior, que en la revisión realizada es la secuela ocular más frecuente. Tampoco figura la erosión corneal recidivante o el menoscabo sufrido en un ojo que ha sido vitrectomizado o fotocoagulado como consecuencia de un desgarro o desprendimiento de retina postraumático y que puede no haber reducido su agudeza visual. Evidentemente, un baremo no puede recoger todas las lesiones posibles, pero sí debería hacerlo con las típicamente postraumáticas para evitar polémicas innecesarias. 


\section{CONCLUSIONES:}

Hemos podido comprobar que los accidentes de tráfico suponen aproximadamente la cuarta parte de las secuelas oculares que vemos en la práctica forense diaria, en una Sección de Oftalmología de una Clínica Médico Forense. Que este tipo de secuelas se producen generalmente como consecuencia de traumatismos cráneo-encefálicos, contusiones oculares directas y esguinces cervicales. La duración media de la baja laboral está en torno a los 7I días, con una indemnización media de 15 puntos, que pueden suponer en un lesionado de una edad entre 20 y 40 años, unos 15.000 euros.

En un porcentaje importante de casos (22\%) no encontramos lesiones objetivas que justifiquen las molestias alegadas por el lesionado, o bien estas no guardan relación evidente con el accidente de tráfico sufrido.

Las secuelas oculares que más habitualmente vemos son: diplopias postraumáticas, disminuciones de la agudeza visual y los casos a los que nos hemos referido anteriormente, de molestias subjetivas que no se justifican por los hallazgos exploratorios encontrados.

Finalmente resaltar que consideramos necesario revisar el actual Baremo de la Ley 34/2003, subsanando los errores y deficiencias a las que nos hemos referido, e incluyendo secuelas oculares típicamente postraumáticas que actualmente no recoge, para facilitar la valoración medico-forense de este tipo de lesionados.

\section{BIBLIOGRAFÍA:}

I. McGwin G, Owsley C. Risk factors for motor vehicle collision-related eye injuries. Arch Ophthalmol. 2005 Jan;I23(I):89-95.

2. Real Decreto Legislativo 8/2004, de 29 de octubre, por el que se aprueba el texto refundido de la Ley sobre responsabilidad civil y seguro en la circulación de vehículos a motor.

3. Karaman K, Gverovic-Antunica A, Rogosic V, Lakos-Krzelj V, Rozga

A, Radocaj-Perko S. Epidemiology of adult eye injuries in SplitDalmatian county. Croat Med J. 2004 Jun;45(3):304-9.

4. Woo JH, Sundar G. Eye injuries in Singapore-don 't risk it. Do mere. A prospective study. Ann Acad Med Singapore. 2006 $0 \mathrm{ct} ; 35(10): 706-18$.

5. Homziuk M, Luksza L. Eye injuries in outpatient clinic of Department of Ophthalmology Medical University Gdansk in 19921993 and 2002-2003 years. Klin Oczna. 2005;107(4-6):247-9.

6.Guly CM, Guly HR, Bouamra 0, Gray rh, Lecky FE. Ocular injuries in pacients with major trauma. Emerg Med J. 2006 Dec;23(I2):915-7. 7. Wang X, Lui X, Wang Y. Analysis on forensic medical appraisals an
VEP characteristic of ocular trauma caused by traffic accidents. Fa Yi Xue za zhi. 2000 May;l6(29:96-8,I28.

8. Esteban de Antonio M. La simulación en ergoftalmología. Madrid: Capta. 1986.

9. Gallego Lago I. Simulación y disimulación en Oftalmología. Barcelona. Glosa SL; 2005.

10. Andonegui Navarro J, Prat Madrazo M. Bilateral traumatic retinopathy associated to whiplash injury. Arch Soc Esp Oftalmol. 2004 Apr;79 (4):185-8.

II. Ley 34/2003 de 4 de noviembre de modificación y adaptación a la normativa comunitaria de la legislación de seguros privados (Tabla VI). Madrid: Mapfre SA; 2004; 7.

12. Menéndez JA. Crítica constructiva a las recientes modificaciones del baremo para la valoración de las secuelas de los accidentes de tráfico. Studium Ophthalmologicum. 2004;|:49-51.

13. Sistema para la valoración de los daños y perjuicios causados a las personas en accidentes de circulación de los vehículos a motor (ley 30/1995). Madrid: Mapfre SA; 2002; 39. 